\title{
NMDA receptor blockade alters the intracellular distribution of neuronal nitric oxide synthase in the superficial layers of the rat superior colliculus
}

\author{
R.E. de Bittencourt-Navarrete, I.C. do Nascimento, M.F. Santiago and \\ R. Mendez-Otero
}

Instituto de Biofísica Carlos Chagas Filho, Universidade Federal do Rio de Janeiro, Rio de Janeiro, RJ, Brasil

Correspondence to: R.E. de Bittencourt-Navarrete, Instituto de Biofísica Carlos Chagas Filho, Universidade Federal do Rio de Janeiro, 21941-590 Rio de Janeiro, RJ, Brasil

E-mail: ruben@biof.ufrj.br

Nitric oxide (NO) is a molecular messenger involved in several events of synaptic plasticity in the central nervous system. Ca ${ }^{2+}$ influx through the N-methyl-D-aspartate receptor (NMDAR) triggers the synthesis of NO by activating the enzyme neuronal nitric oxide synthase (nNOS) in postsynaptic densities. Therefore, NMDAR and nNOS are part of the intricate scenario of postsynaptic densities. In the present study, we hypothesized that the intracellular distribution of nNOS in the neurons of superior colliculus (SC) superficial layers is an NMDAR activity-dependent process. We used osmotic minipumps to promote chronic blockade of the receptors with the pharmacological agent MK-801 in the SC of 7 adult rats. The effective blockade of NMDAR was assessed by changes in the protein level of the immediate early gene NGFI-A, which is a well-known NMDAR activity-dependent expressing transcription factor. Upon chronic infusion of MK-801, a decrease of $47 \%$ in the number of cells expressing NGFI-A was observed in the SC of treated animals. Additionally, the filled dendritic extent by the histochemical product of nicotinamide adenine di-nucleotide phosphate diaphorase was reduced by $45 \%$ when compared to the contralateral SC of the same animals and by $64 \%$ when compared to the SC of control animals. We conclude that the proper intracellular localization of nNOS in the retinorecipient layers of SC depends on NMDAR activation. These results are consistent with the view that the participation of $\mathrm{NO}$ in the physiological and plastic events of the central nervous system might be closely related to an NMDAR activitydependent function.

Key words: Superior colliculus; Nitric oxide synthase; NMDA receptor; Postsynaptic density targeting

Research supported by CAPES Postdoctoral fellowship BEX2856/05-0 to R.E. de Bittencourt-Navarrete, Master fellowship from CNPq to I.C. do Nascimento, financial support from CNPq, grant\#301691/2007-0 and \#473103/2006-0, to R. Mendez-Otero, and by a grant from the Ministry of Science and Technology (MCT) to the Millennium Institute for Tissue Bioengineering, Brazil.

Received April 27, 2008. Accepted January 26, 2009

\section{Introduction}

Much attention has been devoted to elucidating the role of the N-methyl-D-aspartate receptor (NMDAR) in the synaptic plasticity occurring both in the developing and in the mature central nervous system (CNS) of vertebrates. This is a complex and highly regulated ionotropic receptor with conductance for $\mathrm{Na}^{+}, \mathrm{K}^{+}$, and $\mathrm{Ca}^{2+}(1)$. The NMDARmediated $\mathrm{Ca}^{2+}$ influx activates signal transduction cascades that regulate such diverse phenomena as the formation, modification and elimination of synapses (2).

Glutamate receptors are concentrated in the post-synaptic densities (PSD) of the CNS and the effort to characterize constituents of these molecular ensembles has re- 
vealed the presence of key proteins that mediate the targeting of receptors and associated enzymes to the synapse (3). One of these proteins is the PSD protein 95 (PSD-95 or SAP90), a PDZ-domain-containing constituents of glutamate PSDs, which directly associates with NMDAR (4). NMDAR and PSD-95 are both highly concentrated in hippocampal synapses (4).

Like NMDAR and PSD-95, neuronal nitric oxide synthase (nNOS), one of the isoenzymes responsible for the production of nitric oxide (NO), is also localized in the PSDs. nNOS contains a single PDZ domain at its amino terminus, which allows its physical association with PSD95 and, therefore, with the NMDAR (5). NO was first identified as an intercellular messenger molecule in the brain about two decades ago (6). Subsequently, Bredt and Snyder (7) isolated and characterized nNOS. For histological studies the characterization of nNOS cells based on immunohistochemical procedures is considered to be equivalent to the nicotinamide adenine di-nucleotide phosphate diaphorase (NADPH-d) histochemical reaction (8). The physicochemical features of NO such as high diffusibility and the ability to activate various signaling pathways in adjoining cells has been widely recognized. NO is produced in a calcium/calmodulin-dependent fashion from Larginine by the enzyme nNOS (9), and the proximity of the enzyme to the $\mathrm{Ca}^{2+}$ influx provided by the NMDAR is one of the requirements for its catalytic activity (6).

In the developing visual system NOS co-localizes with NMDARs in the rat superior colliculus (SC) (10), and the pharmacological blockade of NMDAR in this nucleus results in the formation of topographically aberrant maps of afferent retinal axons, even before visually driven activity occurs (11). In the chicken optic tectum, the homologue of $\mathrm{SC}$ in mammals, the elimination of transient connections from the ipsilateral retina is dependent on NMDAR stimulation (12).

On the other hand, the role of neural activity in the expression of nNOS is still being elucidated. Previous studies have shown that retinal projections, which are mostly glutamatergic (13), might have a role in the expression or activity of nNOS in the visual system (14). In addition, monocular lid suture results in the abnormal appearance of NADPH-d staining in the lateral geniculate nucleus (15). Furthermore, different nNOS transcripts can arise in pathological conditions of the CNS (16). In the particular case of SC, it is not simple to reconcile the data obtained over the last few years. It has been shown that lack of neural activity produces changes in the intracellular distribution of nNOS in neurons of the SC $(17,18)$. Despite the results of Zhang and co-workers (14) showing that eye removal causes a down-regulation of NADPH-d-positive neurons in the SC, the expression level of the different NADPH-d transcripts seems to be independent of such activity (19).

The observations of Tenorio and co-workers (20) showing that the lack of neural activity arising from the retina, but not from the cortex, is able to modify the intracellular pattern of NADPH-d without a morphological change in SC cells (17) stimulated us to identify which molecular components regulate changes in the intracellular distribution of nNOS. Given the close interaction between NMDAR and nNOS observed in the SC PSDs (21), we asked whether the NMDAR has a direct influence on the intracellular distribution of nNOS in the neurons of SC superficial layers. For this purpose, we promoted a chronic pharmacological blockade of the NMDAR with [(+)-5-methyl-10,11dihydro-5H-dibenzo[a,d]cyclohepten-5,10-imine maleate] (MK-801). The efficiency of blockade with MK-801 was assessed by immunohistochemical reactions that identify changes in the expression of the immediate early gene NGFI-A, which is a marker for NMDAR-mediated activity in the CNS (22). The distribution of nNOS in SC neurons was identified by histochemical reaction with NADPH-d or by immunohistochemistry with specific antibodies directed at nNOS.

\section{Material and Methods}

Animals

Fifteen adult male Lister rats from our breeding colony were housed under conditions of controlled and maintained temperature on a 12:12-h light/dark cycle with food and water available ad libitum. The Committee for the Use of Experimental Animals of our institution approved all experimental protocols applied in this study.

\section{Minipump implantation}

Osmotic minipumps (Alzet model 2002, total capacity $250 \mu \mathrm{L}$, flow rate $0.5 \mu \mathrm{L} / \mathrm{h}$ ) were filled with a solution of 1 $\mathrm{mM}$ MK-801 hydrogen maleate in $0.9 \%$ saline (Research Biochemicals International, USA). This concentration was chosen on the basis of a study using osmotic minipumps, that monitored the minipump release and the concentration reached inside the tissue (23). Male adult rats (3 months old) were anesthetized with an intraperitoneal injection of pentobarbital sodium (35 mg/kg) and placed in a stereotaxic frame. No procedures were started until animals were sufficiently anesthetized, as ascertained by the loss of withdrawal and cornea-blink reflexes. Body temperature, respiratory rate, and anesthesia level were continuously monitored during surgery and additional doses of pentobarbital were given as needed. The skull was surgi- 
cally exposed and a cannula was positioned stereotaxically according to the atlas of Paxinos and Watson (24) (A $-6.3 \mathrm{~mm}, \mathrm{~L}+1.0 \mathrm{~mm}$, from the bregma) in the region just above the left SC. A small craniotomy ( $1 \mathrm{~mm}$ in diameter) was performed and the tip of the cannula was lowered stereotaxically into the cortex to a depth of $3.0 \mathrm{~mm}(\mathrm{~V}+3.0$ $\mathrm{mm}(24))$. The guide catheter and cannula were secured to the skull with cyanoacrylate glue (Super Glue), the minipump was placed subcutaneously in the neck, and the skin was sutured with Prolene Blue monofilament polypropylene sutures (Ethicon, USA). After surgery the animals were kept warm under a hot lamp until recovery and then returned to their cage. Sham surgeries were performed in one group of animals in which a needle was inserted through the cortex following the same stereotaxic coordinates as in the MK-801-treated group. Also, one additional animal was implanted with a saline-filled minipump as part of the control. The infusion of MK-801 was maintained for 10 to 12 days. We chose this period of time based on previous observations from our laboratory showing the requirement of at least 1 week for a consistent change in nNOS immunoreactivity in the SC neurons after eye removal (18).

\section{Perfusions}

Under a regular dark/light cycle (12/12 h), only few NGFI-A-positive cells can be detected relative to those observed after a dark period followed by $2 \mathrm{~h}$ of light exposure (25). Therefore, we placed the animals in a lighttight box for $24 \mathrm{~h}$ the day before sacrifice and then allowed them to receive normal visual stimulation for $2 \mathrm{~h}$. Next, they were anesthetized with $50 \mathrm{mg} / \mathrm{kg}$ sodium pentobarbital and intracardially perfused with $0.9 \%$ saline solution followed by $4 \%$ paraformaldehyde in $100 \mathrm{mM}$ sodium phosphate buffer, $\mathrm{pH}$ 7.4. After dissection, the brains were immersed in $100 \mathrm{mM}$ sodium phosphate buffer containing $20 \%$ sucrose, overnight at $4^{\circ} \mathrm{C}$ and then sectioned at 40 $\mu \mathrm{m}$ on a cryostat (Leica Instruments $\mathrm{GmbH}$, Germany) at $-20^{\circ} \mathrm{C}$ on the coronal planes. Sections containing both hemispheres of the brain were collected in $50 \mathrm{mM}$ Tris buffer, $\mathrm{pH} 7.4$.

\section{Immunochemical and histochemical reactions}

For the immunohistochemical reactions, the sections containing the $\mathrm{SC}$ were incubated as free-floating sections for $1 \mathrm{~h}$ at room temperature with a polyclonal anti-NGFI-A antibody (1:400; Santa Cruz Biotechnology, USA) in a blocking solution containing $5 \%$ normal goat serum and $0.3 \%$ Triton X-100 in 10 mM PBS, pH 7.4, followed by a 2$\mathrm{h}$ incubation in the same solution with a Cy3-conjugated goat anti-rabbit IgG antibody (1:800 dilution, Jackson
ImmunoResearch Laboratories Inc., USA). For double staining, the sections were incubated also with a mouse antinNOS antibody (1:200, Sigma, USA) followed by a goat anti-mouse antibody conjugated with Alexa fluorochrome (1:400 dilution, Jackson ImmunoResearch Laboratories Inc.). The primary antibody was omitted from control sections, which did not show any staining.

For the NADPH-d reactions, sections were collected in $50 \mathrm{mM}$ Tris buffer, $\mathrm{pH} 7.4$, washed twice and reacted as free-floating sections with the following solution: $1 \mathrm{mM} \beta$ NADPH reduced form, $0.5 \mathrm{mM}$ nitroblue tetrazolium dissolved in $100 \%$ dimethylsulfoxide and $1 \%$ Triton X-100 in $50 \mathrm{mM}$ Tris buffer, $\mathrm{pH} 7.4$ (Sigma). The reaction was performed with constant stirring for $1-4 \mathrm{~h}$ at $37^{\circ} \mathrm{C}$. The sections were then washed in Tris buffer, mounted on gelatinized slides, dehydrated with increasing concentrations of alcohol and cover-slipped with Entellan (Merck, Germany).

\section{Data analysis}

NGFI-A. For NGFI-A reactions, tissue sections were analyzed under fluorescent illumination using a rhodamine filter in an Axioplan microscope (Zeiss, Germany). Color images were obtained using a Zeiss AxioCam digital camera connected to this microscope and to a PC computer with the Zeiss Axiovision 3.0 software.

Three coronal sections per animal were selected from rostral, middle and caudal portions of the SC. Three pictures of both hemispheres in each slice, covering almost its entire mediolateral extension, were taken under a 20X objective lens. All NGFI-A-positive cells in each picture were identified, labeled on the computer screen and counted. Absolute numbers of cells were included for comparative analysis. Four groups were used for statistical comparisons: 1) left hemispheres, 2) right hemispheres of control animals, 3) treated hemispheres, and 4) untreated hemispheres of MK-801-infused animals. The cells quantified in each group were compared by one-way multivariate ANOVA followed by the Tukey post hoc multiple comparison test, with $\mathrm{P}<0.05$ as a criterion of statistical significance. Results are reported as means \pm SEM for each group.

$\mathrm{NADPH}-d$. For histological analysis of NADPH-d the same approach of three-field quantification per section in three alternate anteroposterior sessions of the SC recorded under a 20X objective lens was followed, as described above for NGFI-A-labeled cells. The dendritic extensions of all NADPH+ cells in each field were delineated using the morphometric tool of the Zeiss LSM Image Browser. Values of length in micrometers were provided automatically by dragging the cursor from the cell bodies to 
the extremities of every single dendrite. The sum of total dendritic length in each field was divided by the number of cells in order to establish a ratio. Comparisons were performed for the same four groups cited above for NGFI-A. Results are reported as means \pm SEM for each group. Data were analyzed by one-way multivariate ANOVA followed by the Tukey post hoc multiple comparison test, with $\mathrm{P}<0.05$ as a criterion of significant difference.

On the basis of studies performed by our laboratory in the same field (17) and since comparative analysis in-
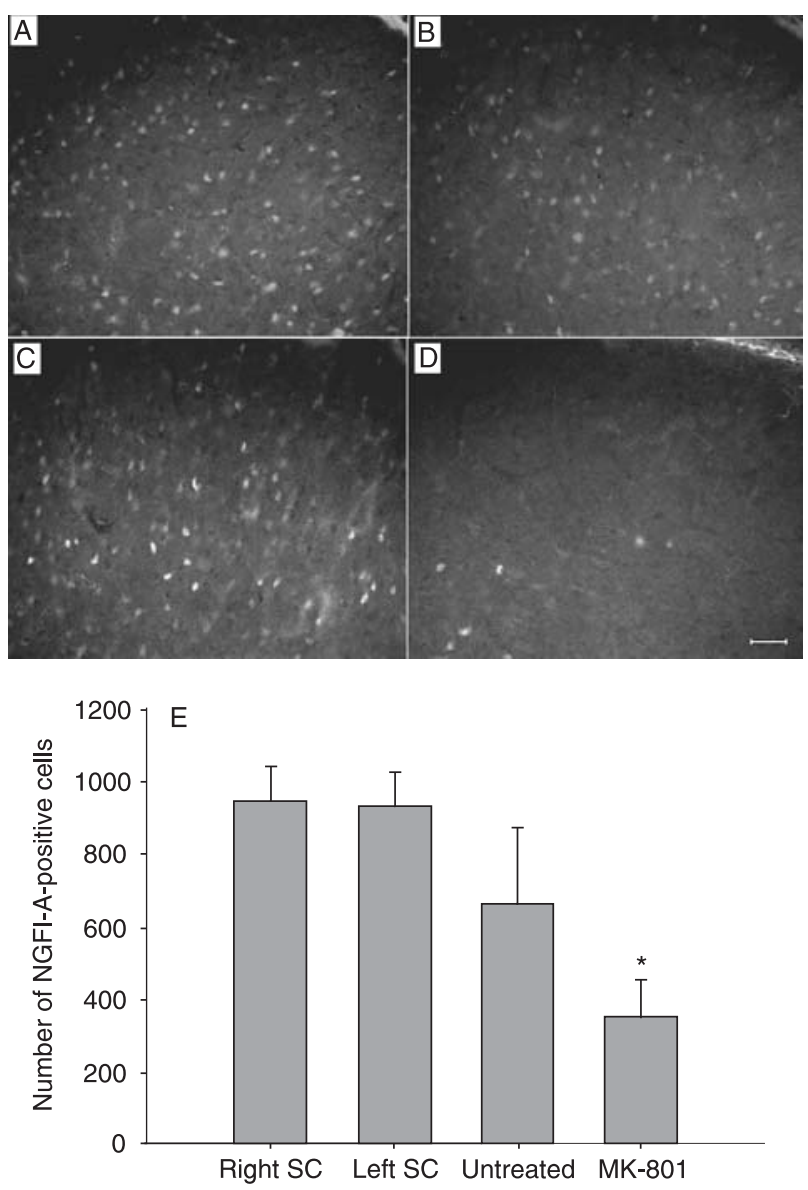

Figure 1. Effect of N-methyl-D-aspartate receptor blockade on NGFI-A expression. Immunohistochemistry performed with antibodies for NGFI-A protein in coronal sections of the right $(A)$ and left (B) superior colliculus (SC) of a normal animal exposed for 2 $\mathrm{h}$ to environmental light after $24 \mathrm{~h}$ of dark rearing. $C$ and $D$ : The same reaction in SC sections of an animal chronically infused with MK-801 for 12 days. The SC under drug infusion is displayed in $D$ and the contralateral $S C$ is shown in $C$. In $E$ a decrease in the number of NGFI-A-positive cells is observed in the MK-801-treated SC. Data are reported as mean \pm SEM for 4 independent experiments. ${ }^{*} P<0.05$ with $F(3,12)=3.960$, oneway multivariate ANOVA followed by the Tukey post hoc multiple comparison test. Magnification bar for all panels $=40 \mu \mathrm{m}$. cluded the two hemispheres of the same animal, stereological procedures were considered to be unnecessary. Moreover, all quantifications were performed twice, first by an aware individual and then in a double-blind mode by another.

The images were analyzed without any modification. Later, for illustration, grayscale conversion and brightness/contrast adjustments were made using the Adobe Photoshop 7.0 software (Adobe, USA). For NADPH-d and double-labeled reactions the images were captured with a LSM 510 META Laser scanning confocal microscope (Zeiss, Germany).

\section{Results}

In the present study, we determined if the blockade of NMDAR with MK-801 interfered with the intracellular distribution of nNOS in neurons of superficial layers of the rat SC. The effectiveness of NMDAR blockade was determined by immunohistochemical reactions with antibodies directed at the NGFI-A protein. The NGFI-A gene codes for a nuclear transcription factor that can be induced by membrane depolarization, thereby allowing its use as an indicator of neuronal activity. In order to block the NMDARmediated activity in the SC, we used osmotic minipumps to infuse MK-801 chronically over a period of ten to twelve days. As stated in the previous section, the time period chosen for MK-801 infusion was based on previous light and electron microscopy observations by our group showing the requirement of at least 1 week for a consistent change in nNOS immunoreactivity in the SC neurons after eye removal (18). To assess changes in the expression level of the NGFI-A gene we performed immunohistochemistry in sections containing the SC. Histological analysis performed for control animals $(\mathrm{N}=4)$ revealed 3728 and $3773 \mathrm{NGFI-A-immunolabeled} \mathrm{cells} \mathrm{in} \mathrm{the} \mathrm{left} \mathrm{and} \mathrm{right}$ hemispheres, respectively. A total of 2618 cells from treated animals $(N=4)$ were counted in the untreated SC and 1377 in the infused SC. Results are reported as means \pm SEM of the total number of cells found per colliculus in each condition, designated as left or right (control colliculi), and untreated or MK-801-treated (infused colliculus). As shown in Figure 1, the infusion of MK-801 led to a drastic reduction in the number of NGFI-A-positive cells in the treated SC. A $47 \%$ decrease in the number of cells expressing $\mathrm{NGFI-A}$ in this hemisphere was observed, indicating an effective blockade of the NMDAR by this pharmacological agent (Figure 1E), which was subsequently reinforced by one-way multivariate ANOVA followed by the Tukey post hoc test, with $\mathrm{F}(3,12)=3.960$ and $\mathrm{P}<0.05$.

To investigate the intracellular distribution of nNOS in 

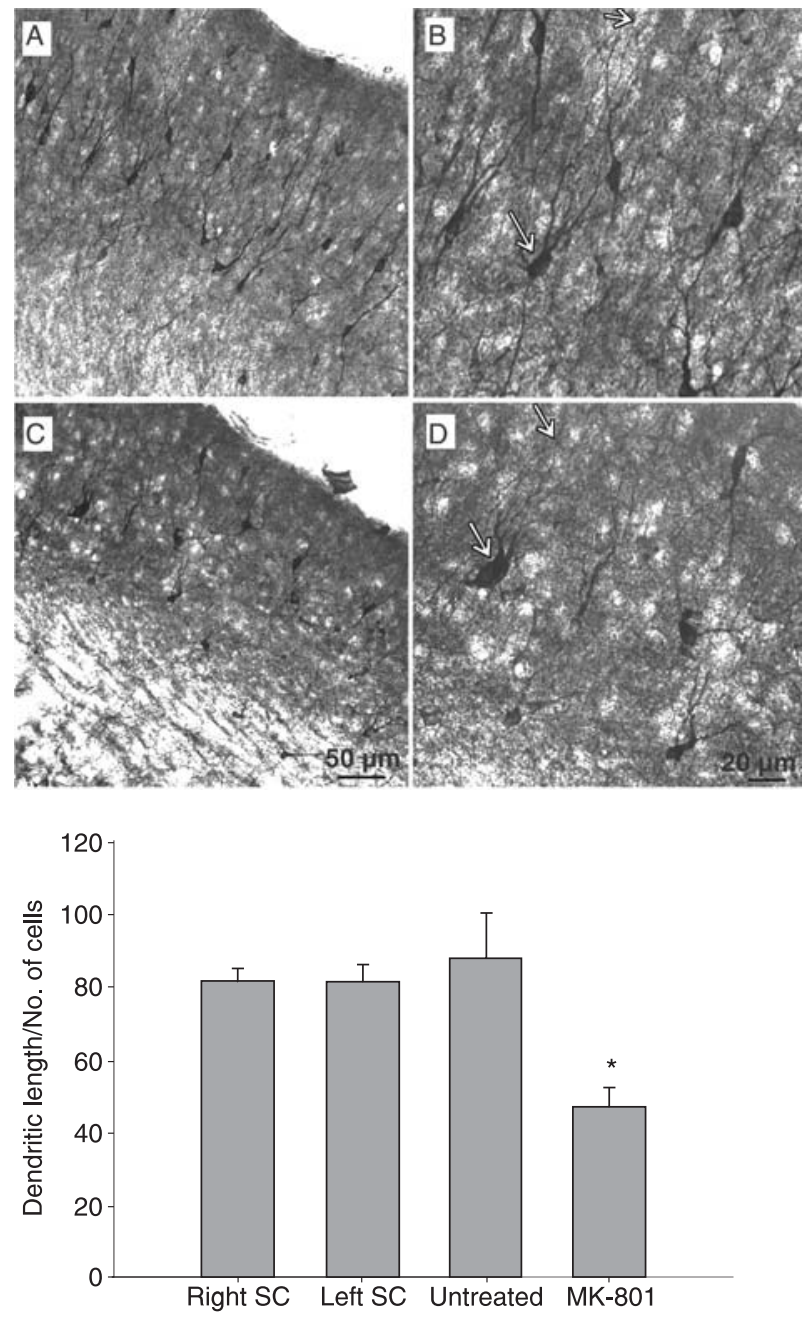

Figure 2. Effect of N-methyl-D-aspartate receptor blockade on the intracellular distribution of neuronal nitric oxide synthase. Top, Photomicrographs of histochemical reaction for nicotinamide adenine di-nucleotide phosphate diaphorase (NADPH-d) in coronal sections through the superficial layers of the superior colliculus (SC). Low (A) and higher (B) magnifications showing the pattern of NADPH-d staining in neurons of an untreated animal. Observe the presence of the NADPH-d histochemistry product delineating cell bodies and the whole extension of dendrites. Low (C) and higher (D) magnifications showing the pattern of NADPH-d neurons of an animal treated with MK-801. Observe the restriction of the reaction product to the soma region and proximal dendrites of the neurons. Arrows in $B$ and $D$ indicate the cell body of representative neurons and the extremity of one of their dendritic branches. E, Quantification of total dendritic length by the number of cells. Significant differences were found between the MK-801-treated SCs and all other conditions, including the contralateral SCs in treated animals $(\mathrm{N}=3$; ${ }^{*} \mathrm{P}<0.05$ with $\mathrm{F}(3,10)=6.496$, one-way multivariate ANOVA followed by the Tukey post hoc multiple comparison test). Magnification bars $=\mathrm{A}$ and $\mathrm{C}, 50 \mu \mathrm{m} ; \mathrm{B}$ and $\mathrm{D}, 20 \mu \mathrm{m}$. the SC we used the histochemical reaction for NADPH-d. Staining for NADPH-d is highly correlated with the presence of nNOS (8). A quantitative analysis revealed a significant difference in the total dendritic tree length of NADPH-d-stained cells as demonstrated by one-way multivariate ANOVA followed by the Tukey post hoc multiple comparison test, with $\mathrm{F}(3,10)=6.496$ and $\mathrm{P}<0.05$ (Figure 2). MK-801 blockade of NMDAR resulted in a $45 \%$ decrease in the total dendritic tree length labeling for NADPH$\mathrm{d}$ in the superficial layers of the SC. This change in the intracellular distribution of nNOS was quantitatively different even when comparing the treated SC to the contralateral one in the same animal, showing a local and specific action of the pharmacological agent. Results are reported as a ratio of dendritic extension to number of cells counted in each field, defined as mentioned in the previous section. The graph in Figure 2 reports the means \pm SEM of these ratios.

Double-labeling immunohistochemistry using two antibodies - anti-nNOS and anti-NGFI-A - confirmed qualitatively the quantitative data shown above (Figure 3 ). The confocal images revealed a drastic reduction in NGFI-A expression as well as in the nNOS-positive immunolabeled dendrites of neurons in the treated SC. In contrast, the $\mathrm{SC}$ of the untreated animals presented numerous NGFI-A-positive cells and a distribution of nNOS clearly spread over the dendritic extension of neurons.

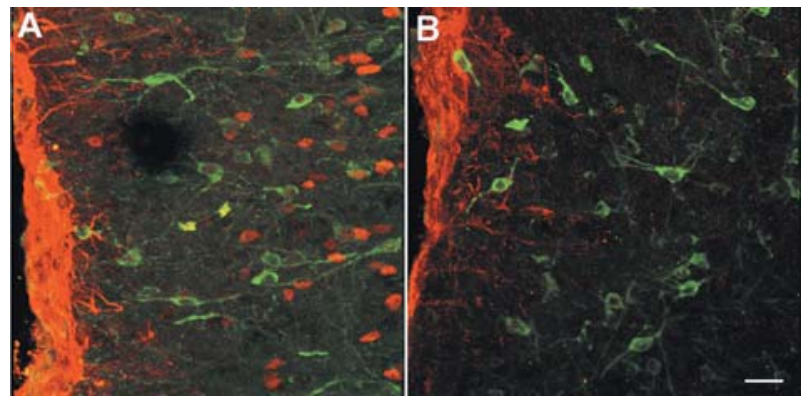

Figure 3. Double staining for NGFI-A and neuronal nitric oxide synthase (nNOS) in the MK-801-treated superior colliculus (SC). Confocal micrographs of representative fields show the double labeling of NGFI-A and nNOS in the SC superficial layers of an untreated $(A)$ and an MK-801-infused animal (B). The immunofluorescence reaction reveals the nNOS labeling (green) and the nuclear localization of NGFI-A (red). In the untreated SC, nNOS labeling reaches distal portions of the dendritic tree and a significant number of NGFI-A-positive nuclei are also present. In the experimental condition, nNOS labeling is restricted around the cell body and virtually no NGFI-A-immunopositive cells appear. Magnification bar for both panels $=20 \mu \mathrm{m}$. 


\section{Discussion}

The NADPH-d reaction identifies all cell types with Golgi staining in the SC (26). The fact that NADPH-d staining is highly correlated with nNOS activity (27) allowed Tenorio and co-workers (17) to use this histochemical reaction to show a strict correlation between the retinal input and the intracellular distribution of nNOS in the retinoreceptive layers of the rat SC. However, these investigators did not clarify whether changes in the intracellular distribution of nNOS were due to a reaction to the optic nerve injury per se or to the removal of visual input. In addition, the role of glutamate receptors in this phenomenon was not tested.

To evaluate the role of NMDAR we used MK-801. The mechanistic functionality of the pharmacological agent used in the present investigation has been extensively studied. The application of NMDA in the presence of MK801 results in complete and irreversible blockade of the NMDAR-mediated excitatory postsynaptic currents (28). In our model, since the retinotectal projection of the animals was intact, the most important requirement for the effective blockade of NMDAR was fulfilled, which is the repeated synaptic stimulation in the presence of MK-801 (29). The developmental changes in NGFI-A expression and its NMDA-mediated induction in the rat SC have already been described by our laboratory (30). We applied a chronic treatment in the SC based on a study demonstrating the rate of release and the concentration achieved in this tissue (23).

Previous investigations have reported the effects of retinal deafferentation on nNOS expression $(14,17,18,20)$. We think it highly unlikely that MK-801 treatment resulted in atrophy of the dendritic tree for the following reasons: 1) it is well established that in the absence of retinal fibers the overall dendritic development is remarkably normal (31). 2) We rely on previous work from our laboratory showing the exact same histochemical pattern for NADPH-d neurons as described here, in the absence of retinal inputs (17). 3) Although the removal of retinal afferents is undoubtedly a more traumatic procedure compared to specific ion channel blockage, it did not change the dendritic morphology of SC neurons (17).

The role of $\mathrm{NO}$ in the nervous system is still poorly understood although, in past years, several studies have been devoted to this issue. Much of the recent knowledge about NO derives from analysis of activity-dependent refinement and maintenance of the topographic retinal projections to the optic tectum (in non-mammalian vertebrates) or to the SC in mammals. In this respect, it is important to point out that the primary stimulus for NO synthesis in CNS neurons is the activation of the NMDAR glutamate receptor. Some studies have shown that NMDAR is important for the mechanisms of retrograde signaling. Nevertheless, it is not clear still if NO participates as a retrograde messenger in the synaptic refinement of the retinotectal system $(32,33)$. It is also known that the maintenance of the topographic map of the SC is disrupted by NMDAR antagonists (34), although knockout of nNOS gene in mice delays but does not prevent the topographical mapping of SC.

According to the results presented here, we can rely upon the premise that, at least in the SC, nNOS is attracted by $\mathrm{Ca}^{2+}$ influx sources. Following activation of $\mathrm{nNOS}$, NO modulates the release of neurotransmitters by presynaptic terminals through a cGMP- and glutamate-dependent mechanism, enhancing their release in some cases, or decreasing it in others (35). Since in the SC nNOS-immunolabeled cells are predominantly inhibitory GABAergic neurons (10), we could speculate that enhancement occurs in this system. In such hypothetical model, as a retrograde messenger, NO would maintain a standard presynaptic glutamate release rate for the arriving retinal terminals. The action of glutamate action on these inhibitory neurons, in turn, would maintain the proper balance between excitatory/inhibitory transmission, stimulating GABAergic release in the local circuitry of the SC.

In spite of the controversy about the role of $\mathrm{NO}$ in retrograde signaling and its importance for the physiology of the nervous system, we demonstrated here how the SC is still an invaluable model for the study of molecular interactions in visual experience-dependent plasticity. In support of this idea, a recent study published by Mu and Poo (36) described the essential role of NMDA and nNOS in the generation of specific patterns of synaptic activity. These investigators demonstrated the dependence on NMDAR and NO for light-induced long-term depression in retinotectal circuits. In this respect, our results provide an interesting clue about the important functional implications of the dynamics of PSD for the physiology of the adult SC. Apparently NMDAR-dependent long-term depression is not induced in adults. Thus, what do the inducers of such phenomena do in the PSD of the rat SC?

Given the recent advances in the understanding of the complexity and dynamics of PSDs, one is induced to think of such molecular assemblies as dynamic structures where proteins can assemble and disassemble constantly. This fundamental feature also implies the existence of targeting mechanisms for the nNOS. The NMDAR-PSD-95-nNOS functional link has been well established as the preferential pathway for NO production in the CNS.

The mechanism by which the inhibition of NMDA trans- 
mission alters nNOS localization probably includes the modification of cytoskeleton structures. This is a reasonable speculation since the association of nNOS with cytoskeleton elements has been well established by our laboratory and others (21). Unfortunately drugs that interfere with cytoskeleton maintenance have dramatic consequences for the physiology of the cell, causing this approach to be broadly disruptive and nonspecific in vivo. A rapid increase of PSD-95 in the synaptoneurosomes and synaptosomes of the rat SC has been shown to occur after a normal retinal stimulus (37). Before eye opening, aggregates of PSD-95 were preferentially localized to cell bodies and proximal dendrites, but $6 \mathrm{~h}$ after eye opening this protein becomes dispersed in the somal and dendritic cytoplasm (37). If we assume that PSD-95 and nNOS are tightly associated in the PSDs it is plausible that the same trafficking mechanisms might be underlying nNOS targeting.

Nevertheless, the role of PSD-95 in synaptic plasticity seems to be more complex and even ubiquitous. In this context, it is possible that PDZ-containing proteins participate indirectly in the maintenance of the PSD molecular assemblies, facilitating signaling events by other enzymes in these microdomains.

It is also interesting to consider some differences between our results and those reported by Yoshii et al. (37).

\section{References}

1. Yamakura T, Shimoji K. Subunit- and site-specific pharmacology of the NMDA receptor channel. Prog Neurobiol 1999; 59: 279-298.

2. Contestabile A. Roles of NMDA receptor activity and nitric oxide production in brain development. Brain Res Brain Res Rev 2000; 32: 476-509.

3. Kennedy MB. The postsynaptic density at glutamatergic synapses. Trends Neurosci 1997; 20: 264-268.

4. Kornau HC, Schenker LT, Kennedy MB, Seeburg PH. Domain interaction between NMDA receptor subunits and the postsynaptic density protein PSD-95. Science 1995; 269: 1737-1740.

5. Brenman JE, Chao DS, Gee SH, McGee AW, Craven SE, Santillano DR, et al. Interaction of nitric oxide synthase with the postsynaptic density protein PSD-95 and alpha1syntrophin mediated by PDZ domains. Cell 1996; 84: 757767.

6. Garthwaite J, Garthwaite G, Palmer RM, Moncada S. NMDA receptor activation induces nitric oxide synthesis from arginine in rat brain slices. Eur J Pharmacol 1989; 172: 413416.

7. Bredt DS, Snyder SH. Isolation of nitric oxide synthetase, a
The time of visual deprivation used by these investigators lasted no more than 4 days while our treatment took at least 10 days. The most noticeable result of the present study is that both procedures, suture and dark rearing although the first allows some degree of retinal-driven input - decrease the synaptic stimulation of NMDAR and $\alpha$ amino-3-hydroxyl-5-methyl-4-isoxazole-propionate (AMPA), while in our model we could assume the maintenance of all glutamate-derived currents but NMDAs. The question of how the interactions between glutamate receptors and PDZ-containing proteins are modulated by synaptic activity to promote the targeting of nNOS in PSDs is still open, and should be addressed in the future in order to clarify fundamental mechanisms of molecular synaptic plasticity.

The present data have shown that a mechanism underlying the proper localization of nNOS requires NMDAR activation and supports the idea that plastic events in the CNS involving NO signaling might be tightly related to NMDAR-dependent function.

\section{Acknowledgments}

The authors thank Tatiana Gonçalves for helpful quantification of the photomicrographs performed in a doubleblind fashion. calmodulin-requiring enzyme. Proc Natl Acad Sci U S A 1990; 87: 682-685.

8. Dawson TM, Bredt DS, Fotuhi M, Hwang PM, Snyder SH. Nitric oxide synthase and neuronal NADPH diaphorase are identical in brain and peripheral tissues. Proc Natl Acad Sci U S A 1991; 88: 7797-7801.

9. Bredt DS, Snyder SH. Nitric oxide: a physiologic messenger molecule. Annu Rev Biochem 1994; 63: 175-195.

10. Soares-Mota M, Henze I, Mendez-Otero R. Nitric oxide synthase-positive neurons in the rat superior colliculus: colocalization of NOS with NMDAR1 glutamate receptor, GABA, and parvalbumin. J Neurosci Res 2001; 64: 501507.

11. Simon DK, Prusky GT, O'Leary DD, Constantine-Paton M. $\mathrm{N}$-methyl-D-aspartate receptor antagonists disrupt the formation of a mammalian neural map. Proc Natl Acad Sci U S A 1992; 89: 10593-10597.

12. Ernst AF, Wu HH, El-Fakahany EE, McLoon SC. NMDA receptor-mediated refinement of a transient retinotectal projection during development requires nitric oxide. J Neurosci 1999; 19: 229-235.

13. Mize RR, Butler GD. Postembedding immunocytochemistry 
demonstrates directly that both retinal and cortical terminals in the cat superior colliculus are glutamate immunoreactive. J Comp Neurol 1996; 371: 633-648.

14. Zhang C, Granstrom L, Wong-Riley MT. Deafferentation leads to a down-regulation of nitric oxide synthase in the rat visual system. Neurosci Lett 1996; 211: 61-64.

15. Gunluk AE, Bickford ME, Sherman SM. Rearing with monocular lid suture induces abnormal NADPH-diaphorase staining in the lateral geniculate nucleus of cats. J Comp Neurol 1994; 350: 215-228.

16. Catania MV, Aronica E, Yankaya B, Troost D. Increased expression of neuronal nitric oxide synthase spliced variants in reactive astrocytes of amyotrophic lateral sclerosis human spinal cord. J Neurosci 2001; 21: RC148.

17. Tenorio F, Giraldi-Guimaraes A, Santos HR, Cintra WM, Mendez-Otero R. Eye enucleation alters intracellular distribution of NO synthase in the superior colliculus. Neuroreport 1998; 9: 145-148.

18. Batista CM, Carneiro $\mathrm{K}$, de Bittencourt-Navarrete RE, Soares-Mota M, Cavalcante LA, Mendez-Otero R. Nitrergic dendrites in the superficial layers of the rat superior colliculus: retinal afferents and alternatively spliced isoforms in normal and deafferented animals. J Neurosci Res 2003; 71: 455-461.

19. de Bittencourt-Navarrete RE, Giraldi-Guimaraes A, Mendez-Otero R. A quantitative study of the neuronal nitric oxide synthase expression in the superficial layers of the adult rat superior colliculus after perinatal enucleation. Int $J$ Dev Neurosci 2004; 22: 197-203.

20. Tenorio F, Giraldi-Guimaraes A, Mendez-Otero R. Removal of the cortical projections alters expression of NOS in the different cell types of the superficial layers of the superior colliculus in rats. An Acad Bras Cienc 2002; 74: 677-681.

21. Batista CM, de Paula KC, Cavalcante LA, Mendez-Otero R. Subcellular localization of neuronal nitric oxide synthase in the superficial gray layer of the rat superior colliculus. Neurosci Res 2001; 41: 67-70.

22. Worley PF, Christy BA, Nakabeppu Y, Bhat RV, Cole AJ, Baraban JM. Constitutive expression of zif268 in neocortex is regulated by synaptic activity. Proc Natl Acad Sci U S A 1991; 88: 5106-5110.

23. Bear MF, Kleinschmidt A, Gu QA, Singer W. Disruption of experience-dependent synaptic modifications in striate cortex by infusion of an NMDA receptor antagonist. $J$ Neurosci 1990; 10: 909-925.

24. Paxinos G, Watson C. The rat brain in stereotaxic coordinates. 2nd edn. Sydney: Academic Press; 1986.

25. Giraldi-Guimaraes A, Mendez-Otero R. Induction of the can- didate-plasticity NGFI-A protein in the adult rat superior colliculus after visual stimulation. Brain Res Mol Brain Res 2005; 133: 242-252.

26. Tenorio F, Giraldi-Guimaraes A, Mendez-Otero R. Morphology of NADPH-diaphorase-positive cells in the retinoceptive layers of the developing rat superior colliculus. Int $J$ Dev Neurosci 1996; 14: 1-10.

27. Hope BT, Michael GJ, Knigge KM, Vincent SR. Neuronal NADPH diaphorase is a nitric oxide synthase. Proc Natl Acad Sci U S A 1991; 88: 2811-2814.

28. Tovar KR, Westbrook GL. Mobile NMDA receptors at hippocampal synapses. Neuron 2002; 34: 255-264.

29. Rosenmund C, Clements JD, Westbrook GL. Nonuniform probability of glutamate release at a hippocampal synapse. Science 1993; 262: 754-757.

30. Giraldi-Guimaraes A, de Bittencourt-Navarrete RE, Nascimento IC, Salazar PR, Freitas-Campos D, Mendez-Otero $R$. Postnatal expression of the plasticity-related nerve growth factor-induced gene $\mathrm{A}$ (NGFI-A) protein in the superficial layers of the rat superior colliculus: relation to $\mathrm{N}$ methyl-D-aspartate receptor function. Neuroscience 2004; 129: 371-380.

31. Sutton JK, Brunso-Bechtold JK. Dendritic development in the dorsal lateral geniculate nucleus of ferrets in the postnatal absence of retinal input: a Golgi study. J Neurobiol 1993; 24: 317-334.

32. Renteria RC, Constantine-Paton M. Nitric oxide in the retinotectal system: a signal but not a retrograde messenger during map refinement and segregation. $J$ Neurosci 1999; 19: 7066-7076.

33. Ruthazer ES, Gillespie DC, Dawson TM, Snyder SH, Stryker MP. Inhibition of nitric oxide synthase does not prevent ocular dominance plasticity in kitten visual cortex. J Physiol 1996; 494 (Part 2): 519-527.

34. Cline HT, Debski EA, Constantine-Paton M. N-methyl-Daspartate receptor antagonist desegregates eye-specific stripes. Proc Natl Acad Sci U S A 1987; 84: 4342-4345.

35. Giraldi-Guimaraes A, Batista CM, Carneiro K, Tenorio F, Cavalcante LA, Mendez-Otero R. A critical survey on nitric oxide synthase expression and nitric oxide function in the retinotectal system. Brain Res Rev 2007; 56: 403-426.

36. Mu Y, Poo MM. Spike timing-dependent LTP/LTD mediates visual experience-dependent plasticity in a developing retinotectal system. Neuron 2006; 50: 115-125.

37. Yoshii A, Sheng MH, Constantine-Paton M. Eye opening induces a rapid dendritic localization of PSD-95 in central visual neurons. Proc Natl Acad Sci U S A 2003; 100: 13341339. 